\title{
Effects of Disorder in Two-Dimensional Photonic Crystal Waveguides
}

\author{
T. N. Langtry, A. A. Asatryan, L. C. Botten \\ CUDOS 83 Department of Mathematical Sciences, \\ University of Technology, Sydney, \\ NSW 2007, Australia
}

\author{
C. M. de Sterke, R. C. McPhedran, P. A. Robinson \\ CUDOS \& School of Physics, \\ University of Sydney, \\ NSW 2006, Australia \\ (Dated: June 11, 2003)
}

\begin{abstract}
The effects of randomness on the guiding properties of waveguides embedded in disordered twodimensional photonic crystals composed of a finite cluster of circular cylinders of infinite length are investigated for TM-polarized radiation. Different degrees of disorder in the radius, filling fraction, refractive index and position are considered for both straight and $90^{\circ}$ bent guides. The crystals exhibit similar sensitivity to refractive index and radius disorder, with a degree of disorder from 15\%-20\% yielding little substantial change in the guiding properties. A smaller range of position disorder is also considered. For strong disorder in radius and refractive index the guide effectively closes. These results were obtained by a Monte Carlo simulation method, and the performance of this method is analyzed. The method requires at least 10 realizations in some cases for convergence to commence; substantially more realizations are required for moderate and strong disorder to achieve accurate results.
\end{abstract}

PACS numbers: 42.25.-p, 41.20.Jb, 41.20.-q, 92.60.Ek

\section{PHOTONIC CRYSTALS}

Photonic crystals - materials with a periodic refractive index distribution - are a subject of worldwide research. One of the main aims is to achieve a photonic band gap - a range of frequencies in which light is unable to propagate in any direction [1-3]. One of the most important properties of such structures is their ability to tailor the path of propagation of light on the scale of the wavelength without diffraction losses (Fig. 1). In particular Mekis et al [4] showed computationally that it is possible to bend the direction of propagation of light by $90^{0}$ without substantial losses - a result that has been confirmed experimentally [5]. Various circuit components which depend on this and related properties, such as $Y$ - [6] and $T$-junctions [1], and channel-drop filters [7], have been proposed.

This unique guiding property of photonic crystals raises the possibility of miniaturizing photonic circuits and, as a consequence, integrating a number of different devices on one substrate to create a full photonic circuit [8]. A model of

(a)

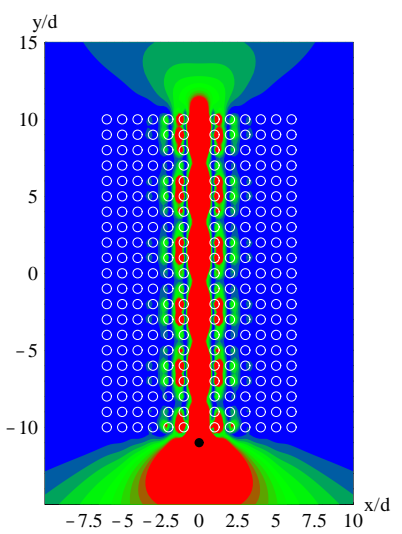

(b)

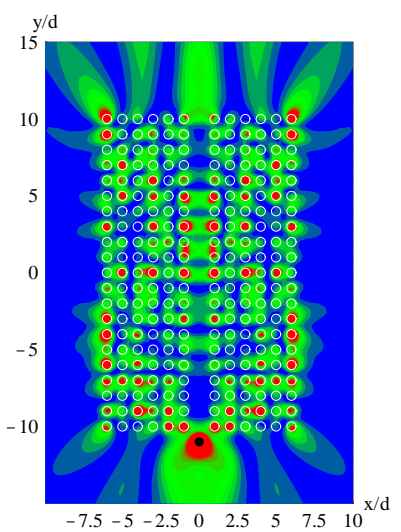

(c)

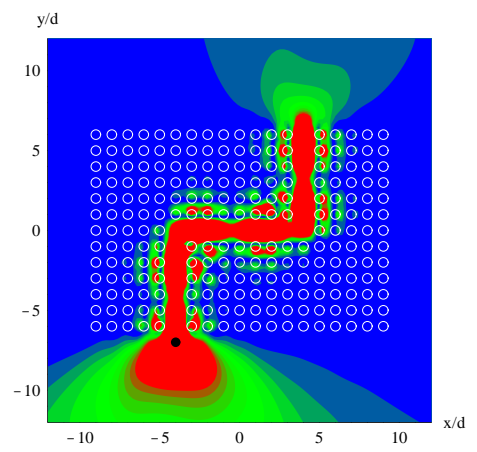

FIG. 1: (Color online) Electric field intensity (normalized to a unit field strength) for a straight guide (252 cylinders) with no disorder and with radius $0.3 d$ at wavelengths: (a) $\lambda=3.3 d$ (in the band gap), (b) $\lambda=5.0 d$ (outside the gap), and (c) guide with bends (226 cylinders with radius $0.3 d$ ) at $\lambda=3.3 d$. The length of this guide (measured down the centre of the channel) is equal to the length of the straight guide. In both cases the guide is excited by a line source parallel to the cylinder axes, as indicated by the black dot. 
an all-optical micro-transistor has already been proposed [9]. To combine and connect the different components inside an all-optical chip it is necessary to establish a clear understanding of the waveguiding properties of such structures. Furthermore, a comprehensive understanding of the tolerances, in other words the degree of imperfection that such structures can withstand, is vital for the effective design of such devices.

Here we investigate the effects of disorder on the waveguiding properties of such structures. We model fabrication defects in two-dimensional waveguides based on a finite cluster of $N_{c}$ dielectric cylinders of infinite length, arranged in a square lattice with lattice constant $d$, by introducing random perturbations in the radii and refractive indices of the cylinders as well as in their positions. The guide is excited by a line source parallel to the cylinders, located close to the entry of the guide. The resulting field intensity and local density of states (LDOS) are calculated using a highly accurate multipole method [10]. Note that in this paper references to the electric field intensity denote the intensity normalized to a unit field strength - the LDOS has been similarly normalized. Quantitative results characterizing the effects of fabrication defects for a TM polarized field are obtained by Monte Carlo simulation [11]. Two aspects are considered: studies of straight guides, and studies of guides containing two $90^{\circ}$ bends. We show that such twodimensional circuits are not very sensitive to disorder, with variations up to $20 \%$ not generally compromising guidance in the cases of radius and refractive index disorder. Disorder of up to $10 \%$ in cylinder positions is also studied, and again we observe that guidance is not compromised. We further study the size of random ensembles needed to establish such tolerances with accuracy, with 10 members at least being necessary to observe commencement of convergence at the classical Monte Carlo rate of $1 / \sqrt{N}$, where $N$ is the number of realizations.

First, we give a short description of the method used to calculate the normalized electromagnetic field (Sec. II) as well as the Monte Carlo simulation method and its convergence. In Sec. III we study the effects of weak disorder of the radius, refractive index and position on guiding for both straight and bent guides, while in Sec. IV we consider the effects of strong disorder and establish the degree of disorder where there is a complete break-down of guidance.

\section{METHOD}

\section{A. Outline of multipole method}

We consider in-plane propagation with the $\mathbf{E}$ field parallel to the cylinders. In this case (the TM polarization) the two-dimensional electric Green tensor contains a single non-trivial component which can be represented using a multipole expansion. The wave equation is solved for a point source $\vec{\nabla}^{2} V\left(\mathbf{r}, \mathbf{c}_{s}\right)+k^{2} n^{2}(\mathbf{r}) V\left(\mathbf{r}, \mathbf{c}_{s}\right)=\delta\left(\mathbf{r}-\mathbf{c}_{s}\right)$ where $V$ is the $z$-component of the electric field due to a source at $\mathbf{c}_{s}$ and normalized to a unit field strength, $k$ is the free-space wavenumber and $n$ is the refractive index. The field may be represented by a Wijngaard expansion, valid everywhere in the crystal matrix [10], given by:

$$
\begin{aligned}
V\left(\mathbf{r}, \mathbf{c}_{s}\right)= & \frac{1}{4 i} \chi^{\operatorname{ext}}\left(\mathbf{c}_{s}\right) H_{0}^{(1)}\left(k\left|\mathbf{r}-\mathbf{c}_{s}\right|\right) \\
& +\sum_{q} \sum_{m=-\infty}^{\infty} B_{m}^{q} H_{m}^{(1)}\left(k\left|\mathbf{r}-\mathbf{c}_{q}\right|\right) e^{i m \arg \left(\mathbf{r}-\mathbf{c}_{q}\right)}
\end{aligned}
$$

where the sums are over rods $(q)$ and cylindrical harmonics $(m)$, the first term represents the source, and the remaining terms represent the field scattered by the cylinders. Here $\chi^{\operatorname{ext}}\left(\mathbf{c}_{s}\right)$ is 1 for a source exterior to all cylinders and 0 otherwise. Similarly, letting $\chi_{\ell}^{\text {int }}\left(\mathbf{c}_{s}\right)$ be 1 if the source lies in cylinder $\ell$ and 0 otherwise, the normalized field expansion inside cylinder $\ell$ with refractive index $n_{\ell}$ is given by

$$
\begin{aligned}
V\left(\mathbf{r} ; \mathbf{c}_{s}\right)= & \frac{1}{4 i} \chi_{\ell}^{\mathrm{int}}\left(\mathbf{c}_{s}\right) H_{0}^{(1)}\left(k n_{\ell}\left|\mathbf{r}-\mathbf{c}_{s}\right|\right) \\
& +\sum_{m=-\infty}^{\infty} C_{m}^{\ell} J_{m}\left(k n_{\ell}\left|\mathbf{r}-\mathbf{c}_{\ell}\right|\right) e^{i m \arg \left(\mathbf{r}-\mathbf{c}_{\ell}\right)}
\end{aligned}
$$

A local expansion is also valid in an annulus outside each ( $\ell$ 'th) cylinder:

$$
\begin{aligned}
V\left(\mathbf{r}, \mathbf{c}_{s}\right)=\sum_{m=-\infty}^{\infty} & {\left[A_{m}^{\ell} J_{m}\left(k\left|\mathbf{r}-\mathbf{c}_{l}\right|\right)\right.} \\
& \left.+B_{m}^{\ell} H_{m}^{(1)}\left(k\left|\mathbf{r}-\mathbf{c}_{l}\right|\right)\right] e^{i m \arg \left(\mathbf{r}-\mathbf{c}_{l}\right)} .
\end{aligned}
$$


These expressions for $V$ must be consistent, and Graf's addition theorem is used to equate (1) and (3), given their use of different origins. We thus obtain [12] an identity of the form $A_{m}^{\ell}=\chi^{\operatorname{ext}}\left(\mathbf{c}_{s}\right) K_{m}^{\ell}+\sum_{q \neq \ell} \sum_{m} S_{m p}^{\ell q} B_{p}^{q}$ or, in matrix form,

$$
\mathbf{A}=\chi^{\operatorname{ext}}\left(\mathbf{c}_{s}\right) \mathbf{K}+\mathbf{S B}
$$

where $\mathbf{K}$ is associated with the source and $\mathbf{S B}$ is due to scattering by all cylinders $q \neq \ell$. An additional relation between $\mathbf{A}$ and $\mathbf{B}$ is needed to solve this system; this is obtained from the boundary conditions at each cylinder boundary and reads

$$
\mathbf{B}=\mathbf{R A}+\mathbf{T} \chi^{\mathrm{int}} \mathbf{Q}
$$

where $\mathbf{R}$ and $\mathbf{T}$ denote matrices of cylindrical harmonic reflection and transmission coefficients [13] and the term $\mathbf{Q}$ represents a source interior to a cylinder. A full derivation may be found in [10]. Combining (4) and (5) yields the equation

$$
(\mathbf{I}-\mathbf{R S}) \mathbf{B}=\chi^{\mathrm{ext}} \mathbf{R K}+\chi^{\mathrm{int}} \mathbf{T} \mathbf{Q}
$$

This system is then solved to find the coefficients $B_{m}^{\ell}$ and thus the entire field external to the cylinders. Reconstruction of the field inside the $\ell^{\prime}$ th cylinder requires the coefficients $C_{m}^{\ell}$. Again these are obtained from boundary conditions, yielding (in matrix form)

$$
\mathbf{C}=\mathbf{T}^{\prime} \mathbf{A}+\mathbf{R}^{\prime} \chi^{\text {int }} \mathbf{Q}
$$

where $\mathbf{R}^{\prime}$ and $\mathbf{T}^{\prime}$ are matrices of reflection and transmission coefficients. The method is efficient and accurate, even for disordered structures in which the positions, radii and refractive indices of the cylinders are arbitrary.

A key quantity characterizing the radiation dynamics of a fluorescent source is the (spatially resolved) local density of states [14] $\tilde{\rho}(\mathbf{r} ; \omega)$, given for $\mathbf{r}$ in the matrix by

$$
\tilde{\rho}(\mathbf{r} ; \omega)=-\frac{2 \omega}{\pi c^{2}} \Im[V(\mathbf{r}, \mathbf{r} ; \omega)]
$$

where $\Im$ denotes the imaginary part. The LDOS provides the spectral distribution of modes to which a source at a particular point may couple and is proportional to the total power emitted by a line source at that point. Thus for a given frequency a large value of $\tilde{\rho}$ indicates that light emission at that frequency is enhanced-correspondingly, small $\tilde{\rho}$ indicates suppression of emission. In the following we normalize $\tilde{\rho}$ to the dimensionless quantity $\rho=\tilde{\rho} \pi c^{2}(2 \omega)^{-1}$.

\section{B. Monte Carlo simulation}

We consider ensembles of realizations with random perturbations in the position $\mathbf{r}_{\ell}$, radius $a_{\ell}$ or index $n_{\ell}$ of each cylinder. For studies of intensity in the straight guide we consider five types of perturbations:

R1: (Uniform position-two-sided) $\mathbf{c}_{\ell}$ uniformly distributed with $\mathbf{c}_{\ell}^{(0)} / d-\delta_{\mathbf{c}} \leq \mathbf{c}_{\ell} / d \leq \mathbf{c}_{\ell}^{(0)} / d+\delta_{\mathbf{c}}$, for vector perturbations $\delta_{\mathbf{c}}=(0.033,0.033),(0.067,0.067),(0.1,0.1)$. Here $\mathbf{c}_{\ell}^{(0)}$ denotes the unperturbed position of the $\ell$ 'th cylinder as indicated in Fig 1.

A1: (Uniform radius-one-sided) $a_{\ell} / d$ uniformly distributed with $0.3 \leq a_{\ell} / d \leq 0.3+\delta_{a}$, for $\delta_{a}=0.01, \ldots, 0.12$ in 12 steps.

A2: (Uniform radius - two-sided) $a_{\ell} / d$ uniformly distributed with $0.3-\delta_{a} \leq a_{\ell} / d \leq 0.3+\delta_{a}$, for $\delta_{a}=0.01, \ldots, 0.12$ in 12 steps.

A3: (Uniform area-two-sided) cylinder cross-sectional area $A_{\ell}$ uniformly distributed with $\pi 0.3^{2}-\delta_{A} \leq A_{\ell} / d^{2} \leq$ $\pi 0.3^{2}+\delta_{A}$ with maximal area perturbation $\delta_{A}=\pi\left(2 \delta_{a}+\delta_{a}^{2}\right)$ determined by choosing $\delta_{a}=0.01, \ldots, 0.12$ in 12 steps.

N1: (Uniform index-two-sided) $n_{\ell}$ uniformly distributed with $3.0-\delta_{n} \leq n_{\ell} \leq 3.0+\delta_{n}$, for $\delta_{n}=0.1, \ldots, 1.2$ in 12 steps.

In studies of the intensity along the bent guide we considered cases R1, A1, A3 and three instances of N1: $\delta_{n}=0.3$, $0.6,1.0$. We also studied the effects of disorder on the local density of states (LDOS) in selected cases.

The simulation program was implemented as a Fortran95 parallel program using a master/slave configuration, with slave processors handling individual realizations. The computational work was carried out on an SGI Origin 
2400 shared-memory parallel machine using MPI to support multiple slave processes and OpenMP to support loop parallelism within a slave process, and on a Compaq Alphaserver SC distributed-memory parallel machine using only MPI.

Initial studies on the SGI Origin consisted of ensembles of 50 realizations, with fields computed at points on a two-dimensional grid. The same ensemble size was used for early studies on the Alphaserver SC. Changes in the scheduling algorithm used on the latter machine prompted a reduction in the ensemble size to 48 realizations in later studies. However, this had a negligible effect on the results. We investigate the relationship between the ensemble size and computational accuracy below, using additional results from studies using larger ensembles, but with fields computed at selected points.

\section{Convergence}

Our computational objective was to estimate the expectation values of the normalized intensity $E\left(|V|^{2}\right)$ or the normalized LDOS $E(\rho)$ at each point $\mathbf{r}$ on a grid $\mathcal{P}\left(301 \times 301\right.$ points for $|V|^{2}$ and $151 \times 151$ for $\left.\rho\right)$. The multipole method (Sec. II A) is exact up to the truncation of the series - hence the Monte Carlo method estimates the mean of the truncated series. Some indication of the truncation error is found by considering the difference in the results obtained for a single crystal with no disorder using additional terms in the expansion (1)-(3). For the straight guide, the average relative difference observed at grid points within the cluster between the normalized intensity results using 9 terms $\left(-4 \leq m \leq 4\right.$ in (1)-(3)) and 11 terms $(-5 \leq m \leq 5)$, that is $\left\langle\left.|| V_{11}\right|^{2}(\mathbf{r} ; \omega)-\left.\left|V_{9}\right|^{2}(\mathbf{r} ; \omega)|/| V_{11}\right|^{2}(\mathbf{r} ; \omega)\right\rangle$, was $2.1 \times 10^{-4}$, with the maximum relative difference being $1.7 \times 10^{-2}$.

The Monte Carlo method is subject to additional error due to estimating the true (population) mean by an ensemble average (sample mean). This is the dominant source of error, and to monitor the convergence of the process we computed the standard error at each grid point $\mathbf{r} \in \mathcal{P}$. We note (see [11] for example) that the standard error is simply the standard deviation of the distribution of the sample mean about the population mean, and may be estimated in terms of the standard deviation $s_{N}$ of the individual values in the ensemble. In particular, let $N$ be the number of realizations and let $\left|V_{j}\right|^{2}(\mathbf{r} ; \omega)$ be the normalized intensity and $\rho_{j}(\mathbf{r} ; \omega)$ be the normalized LDOS for the $j$ 'th realization. For convenience, let $v_{j}$ denote either $\left|V_{j}\right|^{2}(\mathbf{r} ; \omega)$ or $\rho_{j}(\mathbf{r} ; \omega)$ as appropriate. Then for fixed $\mathbf{r}, \omega$ the variables $v_{1}, \ldots, v_{N}$ are independent and identically distributed random variables. Let $E(v)$ and $\sigma_{v}^{2}$ respectively denote the corresponding mean and variance. If these are finite then the standard error is

$$
\begin{aligned}
{\left[\operatorname{Var}\left(E(v)-\frac{1}{N} \sum_{i=1}^{N} v_{i}\right)\right]^{1 / 2} } & =\left[\frac{1}{N^{2}} \operatorname{Var}\left(\sum_{i=1}^{N} v_{i}\right)\right]^{1 / 2} \\
& =\left(\frac{1}{N^{2}} N \sigma_{v}^{2}\right)^{1 / 2}=\frac{\sigma_{v}}{\sqrt{N}} .
\end{aligned}
$$

According to the Central Limit Theorem, under these assumptions the limiting distribution (as $N \rightarrow \infty$ ) of the sample mean is normal. Estimating $\sigma_{v}$ by the sample variance then lets us determine confidence intervals for $E(v)$ in particular, a $68.3 \%$ confidence interval for the mean normalized intensity is approximately $\left(\langle v\rangle-\frac{\sigma_{N}}{\sqrt{N}},\langle v\rangle+\frac{\sigma_{N}}{\sqrt{N}}\right)$, that is, $68.3 \%$ of samples of this size capture the expected normalized intensity in this interval. Correspondingly, a $95.4 \%$ confidence interval on $E(v)$ is given by $\left(\langle v\rangle-\frac{2 \sigma_{N}}{\sqrt{N}},\langle v\rangle+\frac{2 \sigma_{N}}{\sqrt{N}}\right)$. As the sample size $N \rightarrow \infty$ the interval width decreases as $1 / \sqrt{N}$ in both cases.

In addition to computing the standard error at individual grid points we studied the convergence of the method by tracking the convergence of the standard error $S_{N}\left(\phi_{v}\right)$ of the norm of the vector of normalized intensity (or LDOS) values over the grid, that is, of

$$
\begin{aligned}
\phi_{V}(j) & \stackrel{\text { def }}{=}\left[\left.\left.\sum_{\mathbf{r} \in \mathcal{P}}|| V_{j}\right|^{2}(\mathbf{r} ; \omega)\right|^{2}\right]^{1 / 2}, \\
\phi_{\rho}(j) & \stackrel{\text { def }}{=}\left[\sum_{\mathbf{r} \in \mathcal{P}}\left|\rho_{j}(\mathbf{r} ; \omega)\right|^{2}\right]^{1 / 2},
\end{aligned}
$$

for $1 \leq j \leq N$. Figure 2 plots the estimated standard error $S_{N}\left(\phi_{V}\right)$ against $N$ for the straight guide in case A1 (uniform radius, one-sided) for three levels of disorder. In all three cases we observe an initial increase in $S_{N}\left(\phi_{V}\right)$. This is due to the large variation in a sample average for a small sample size. As the sample size increases this variability 


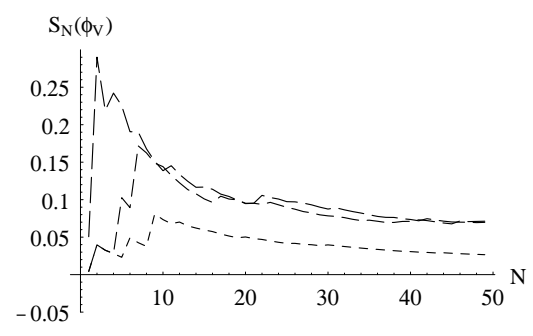

FIG. 2: Convergence of Monte Carlo simulations in case A1: standard error $S_{N}\left(\phi_{V}\right)$ vs. number $N$ of realizations, for (in order of increasing dash length) $\delta_{a}=0.03$ (10\% randomization), 0.06 (20\% randomization) and 0.1 (33.3\% randomization). Note the initial increases for small $N$.
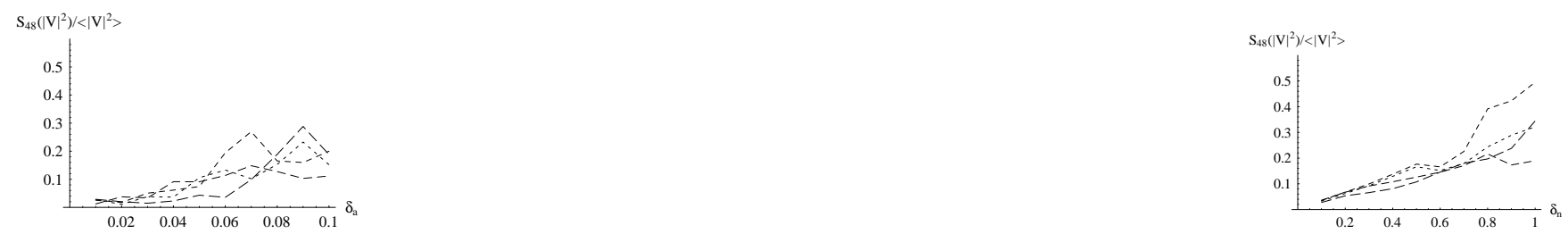

FIG. 3: Relative standard error $S_{48}\left(|V|^{2}\right) /\left\langle|V|^{2}\right\rangle$ after 48 realizations for straight guide vs. level of disorder in cases A1 (left) and N1 (right) at points in the crystal (in order of increasing dash length): at the midpoint of the guide, a point in the middle of the left half of the crystal matrix, the guide entrance, and the guide exit.

peaks, after which we observe convergence setting in, albeit somewhat later $(N \approx 10)$ for weak disorder than for strong disorder $(N \approx 4)$. Ignoring this initial phase and fitting the remaining data $(N>10)$ to the expression $c N^{b}$ yields $b=-0.68,-0.46$, and -0.48 respectively, these values being consistent with the expected rate of convergence of $N^{-0.5}$.

Within the cluster the standard error varies with both position and level of disorder. Figure 3 illustrates the relative standard error $S_{N}\left(|V|^{2}\right) /\left\langle|V|^{2}\right\rangle$ after $N=48$ realizations at each of 4 points in the cluster as a function of the level of disorder, for the straight guide in cases A1 and N1. Strong disorder is characterized by large variations in crystal structure between different realizations, yielding higher standard error in the Monte Carlo estimates of mean field values than for weak disorder.

As we observe in Sec. III, guiding is preserved for disorder in radius and index of up to approximately $20 \%$ - that is, $\delta_{a} \leq 0.06$ and $\delta_{n} \leq 0.6$. Fig. 2 suggests that, in the region where guiding is preserved, more than 10 realizations are required to achieve reliable estimates of the expected normalized intensity or LDOS. For precision, Table I gives the minimum sample size, equal to or exceeding 10, required at each level of disorder examined in cases A1 and N1 to achieve confidence intervals at selected levels for $10 \%$ error in the estimated mean normalized intensity (that is, $|E(v)-\langle v\rangle| /\langle v\rangle<0.1)$ at the point $(0,0)$, in the middle of the straight guide.

\section{ONE-DIMENSIONAL BEHAVIOUR FOR SMALL DISORDER}

First we consider how, for straight guides, the guiding characteristics vary with increasing disorder in given parameters. Figure 4 gives thumbnail illustrations of normalized intensity maps over representative individual crystal realizations in case A1. Figure 5 gives corresponding histograms of the normalised intensity attenuation coefficient $\alpha d$ (where $-\alpha d$ is the slope of a straight line fitted to the graph of the natural log of normalized intensity versus normalized distance $y / d$ along the centre of the guide). We observe that guiding is still strong at $10 \%$ and even $20 \%$ disorder, but that the channel is effectively closed at $33.3 \%$ disorder.

Table II gives the estimated means and standard deviations of the sampled normalized intensity attenuation coefficients in cases R1, A3 and N1. We note here that the expectation value of the fill fraction of the crystal coincides with the fill fraction of the unperturbed crystal, whereas in cases A1 and A2 it does not. We discuss this point in more detail below. The results presented in Table II indicate that very similar behaviour occurs in all three cases for small disorder, and in cases A3 and N1 for large disorder. In particular, in cases A3 and N1 we observe an apparently linear relationship between $\delta$ and $\sigma_{|V|^{2}}$ for small disorder $\left(\delta_{a} \leq 0.06\right.$ and $\left.\delta_{n} \leq 0.6\right)$. 


\begin{tabular}{r|lll|lrr|llll}
\hline & \multicolumn{3}{|c|}{$\mathrm{R} 1$} & \multicolumn{5}{|c|}{$\mathrm{A} 3$} & \multicolumn{4}{c}{$\mathrm{N} 1$} \\
$N$ & $\delta_{\mathbf{c}}$ & $68.3 \%$ & $95.4 \%$ & $\delta_{a}$ & $68.3 \%$ & $95.4 \%$ & $\delta_{n}$ & $68.3 \%$ & $95.4 \%$ \\
\hline 48 & 0.033 & 17 & - & 0.01 & 10 & 10 & 0.1 & 10 & 11 \\
48 & 0.067 & 38 & - & 0.02 & 10 & 15 & 0.2 & 10 & - \\
60 & 0.1 & 55 & - & 0.03 & 12 & 51 & 0.3 & 44 & - \\
90 & & & & 0.04 & 21 & 77 & 0.4 & 82 & - \\
200 & & & & 0.05 & 52 & 183 & 0.5 & 97 & - \\
200 & & & & 0.06 & 40 & 164 & 0.6 & - & - \\
228 & & & & 0.07 & 51 & 178 & 0.7 & 119 & - \\
228 & & & & 0.08 & 80 & - & 0.8 & - & - \\
228 & & & & 0.09 & 162 & - & 0.9 & - & - \\
228 & & & & 0.10 & 140 & - & 1.0 & - & - \\
228 & & & & 0.11 & 205 & - & 1.1 & - & - \\
228 & & & & 0.12 & - & - & 1.2 & - & -
\end{tabular}

TABLE I: Number of realizations equal to or exceeding 10 at which $68.3 \%$ confidence intervals and $95.4 \%$ confidence intervals for a maximum relative error of $10 \%$ in the estimated mean normalized intensity at the origin (centre of the guide) were first achieved. Column $1(N)$ gives the total number of realizations considered. The remaining three columns give the results for cases R1 (left), A3 (centre) and N1 (right). A dash indicates that the confidence level was not achieved. In each row the perturbation parameter $\delta$ has the same magnitude relative to the corresponding unperturbed parameter (respectively, $d=1$, $a / d=0.3$ and $n=3.0)$.

\begin{tabular}{ccc|ccc|ccc}
\hline \multicolumn{3}{c|}{$\mathrm{R} 1$} & \multicolumn{4}{|c|}{$\mathrm{A} 3$} & \multicolumn{3}{|c}{$\mathrm{N} 1$} \\
$\delta_{\mathbf{c}}$ & $\langle\alpha d\rangle$ & $\sigma_{\alpha d}$ & $\delta_{a}$ & $\langle\alpha d\rangle$ & $\sigma_{\alpha d}$ & $\delta_{n}$ & $\langle\alpha d\rangle$ & $\sigma_{\alpha d}$ \\
\hline 0.033 & 0.014 & 0.011 & 0.01 & 0.014 & 0.0084 & 0.1 & 0.014 & 0.010 \\
0.066 & 0.019 & 0.022 & 0.02 & 0.017 & 0.017 & 0.2 & 0.016 & 0.021 \\
0.10 & 0.028 & 0.033 & 0.03 & 0.020 & 0.026 & 0.3 & 0.020 & 0.031 \\
& & & 0.04 & 0.026 & 0.031 & 0.4 & 0.029 & 0.042 \\
& & & 0.05 & 0.035 & 0.039 & 0.5 & 0.042 & 0.056 \\
& & & 0.06 & 0.046 & 0.054 & 0.6 & 0.059 & 0.073 \\
& & & 0.07 & 0.064 & 0.070 & 0.7 & 0.084 & 0.094 \\
& & & 0.08 & 0.10 & 0.084 & 0.8 & 0.12 & 0.11 \\
& & 0.09 & 0.20 & 0.13 & 0.9 & 0.21 & 0.15 \\
& & 0.1 & 0.33 & 0.18 & 1.0 & 0.30 & 0.18
\end{tabular}

TABLE II: Estimated means $\langle\alpha d\rangle$ and standard deviations $\sigma_{\alpha d}$ of the distributions of normalized intensity attenuation coefficients $\alpha d$ in cases R1, A3 and N1.

Figure 6 shows maps of the mean normalized intensity (over 50 realizations) for case A1 in the straight (left) and bent (middle) guides at the same levels of randomization as seen in Fig. 4. We observe similar qualitative behaviour in both cases. The rightmost column of Fig. 6 shows the mean LDOS mapped over the crystal with the bent guide. For $10 \%$ randomization we observe very small LDOS values in the interior of the crystal, with relatively large values along the guide and at the crystal surface, indicating that radiation is suppressed in the crystal except along the guide. The left and middle columns of Fig. 6 indicate that, with the source located at the entrance to the guide, the propagating state along the guide is excited in both straight and bent guides.

As disorder increases, the LDOS maps in Fig. 6 indicate that the available states in the crystal increase until, at $33.3 \%$, there are similar numbers of states available for radiation into the crystal interior and radiation along the guide. The mean normalized intensity map illustrates the consequent loss of guiding. Similar results obtain for disorder in the refractive indices of the cylinders: guiding is maintained at levels of up to $20 \%$ randomization of the indices, after which a progressive loss of guiding is observed.

The straight and bent guides have different geometries, but are of the same length when measured along the centre of the channel. Figure 7 superimposes logarithmic plots of mean normalized intensity along the guide for bent and straight guides at the same levels of randomization in case A1. The spike indicates the source position, and vertical lines indicate the beginning and end of the channel. There is excellent agreement between straight and bent guides for $\delta_{a} \leq 0.06$ (i.e. $20 \%$ disorder), but differences begin to emerge for $\delta_{a}>0.06$. This suggests that the two guides demonstrate essentially one-dimensional behaviour for weak disorder. To first order, the two guides appear very similar in this regime - both demonstrate Fabry-Perot beating with comparable beat lengths. For higher degrees of disorder this close agreement breaks down. However, when the plots for various $\delta_{a}$ along the centre of just the straight guide are superimposed (Fig. 8) we note a significant variation in beat lengths as $\delta_{a}$ increases, although the phase jump upon reflection at the far end of the channel occurs appears to be constant. 


$$
\delta_{a}=0.03(10 \% \text { randomisation) }
$$
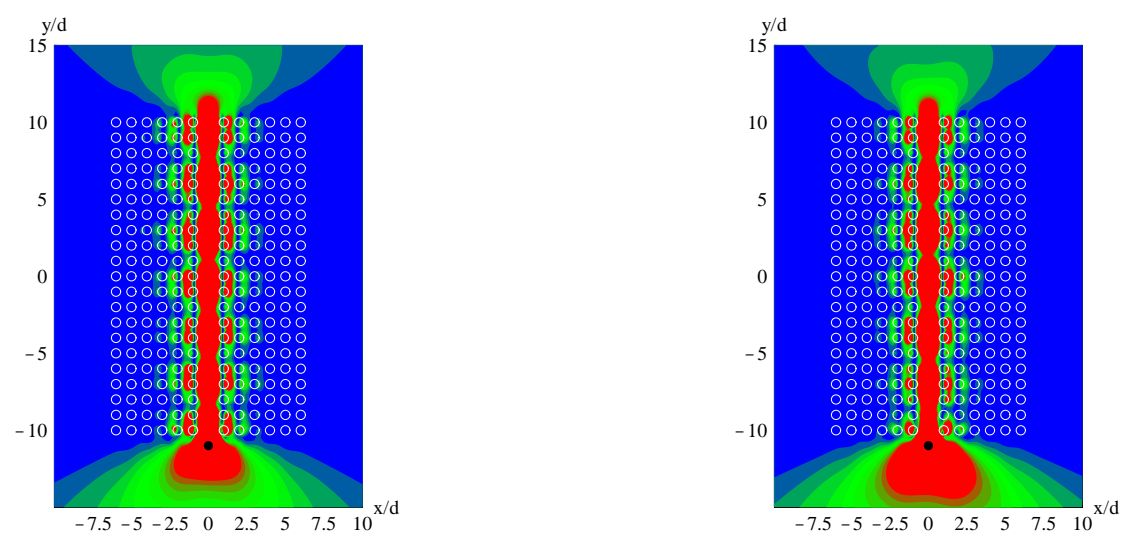

$\delta_{a}=0.06(20 \%$ randomisation)
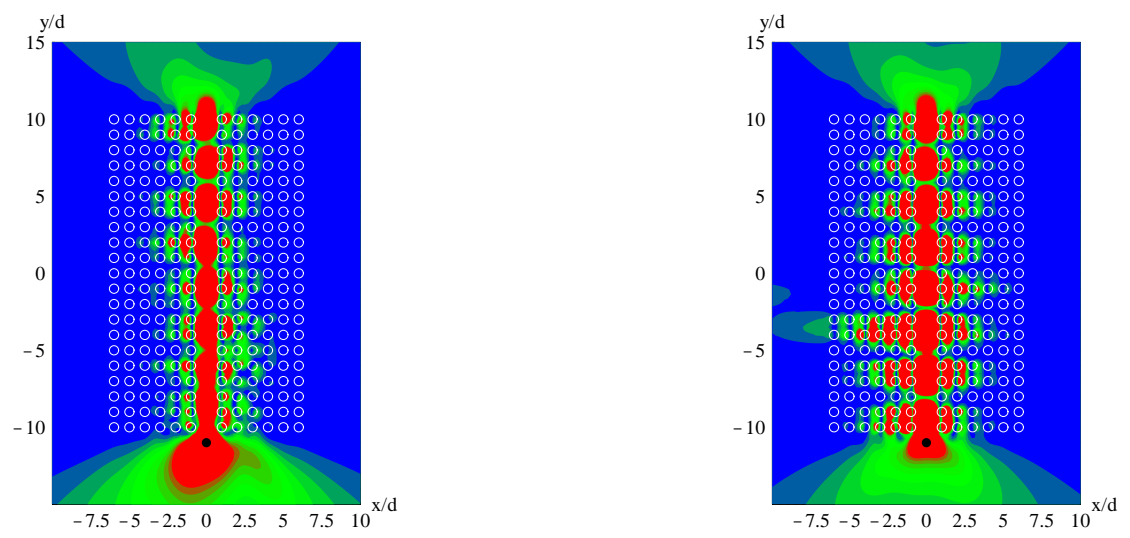

$\delta_{a}=0.10(33.3 \%$ randomisation $)$
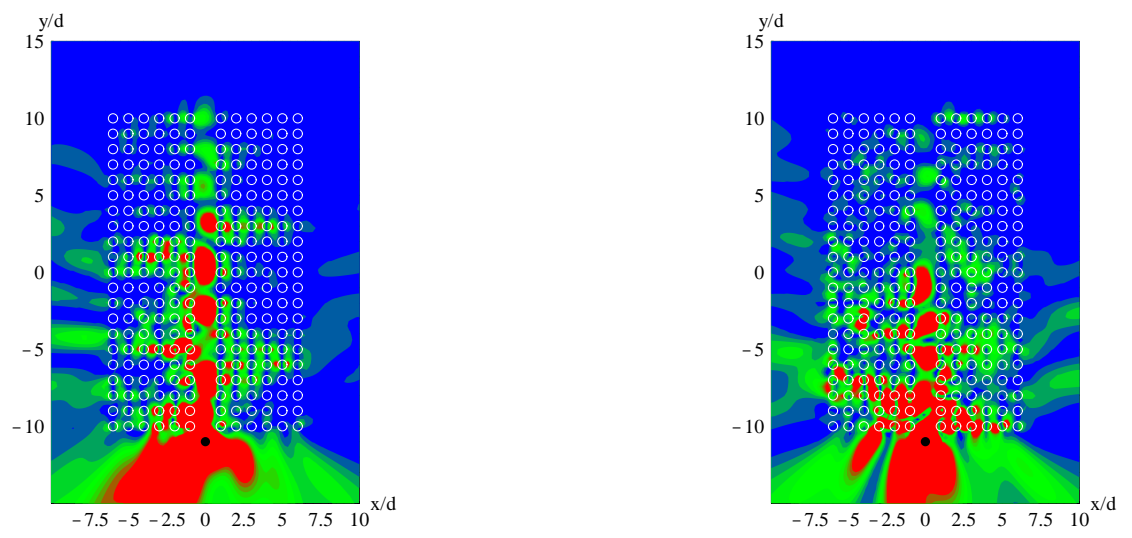

FIG. 4: (Color online) Normalized intensity in individual crystal realizations of straight guide with disordered radius: one-sided perturbations of normalized radius $a / d$ uniformly distributed on $\left[0, \delta_{a}\right]$.

Since in case A1 the normalized cylinder radius $a_{\ell} / d$ is uniformly distributed on $\left[0.3,0.3+\delta_{a}\right]$, changing $\delta_{a}$ results in a change in the mean cross-sectional area of the cylinders and hence in the fill-fraction of the crystal, giving rise to changes in the optical properties, such as the beat length, of the bulk crystal. To demonstrate this, we computed the beat frequency, as a function of radius, for an ideal waveguide which is modeled as a cavity of arbitrary width $h$ sandwiched between two identical semi-infinite photonic crystals. Each of the crystals can be considered as an infinite stack of cylinder gratings of period $d$, satisfying a Bloch condition with Bloch factor $\exp \left(i \beta_{0} d\right)$. Using a transfer 

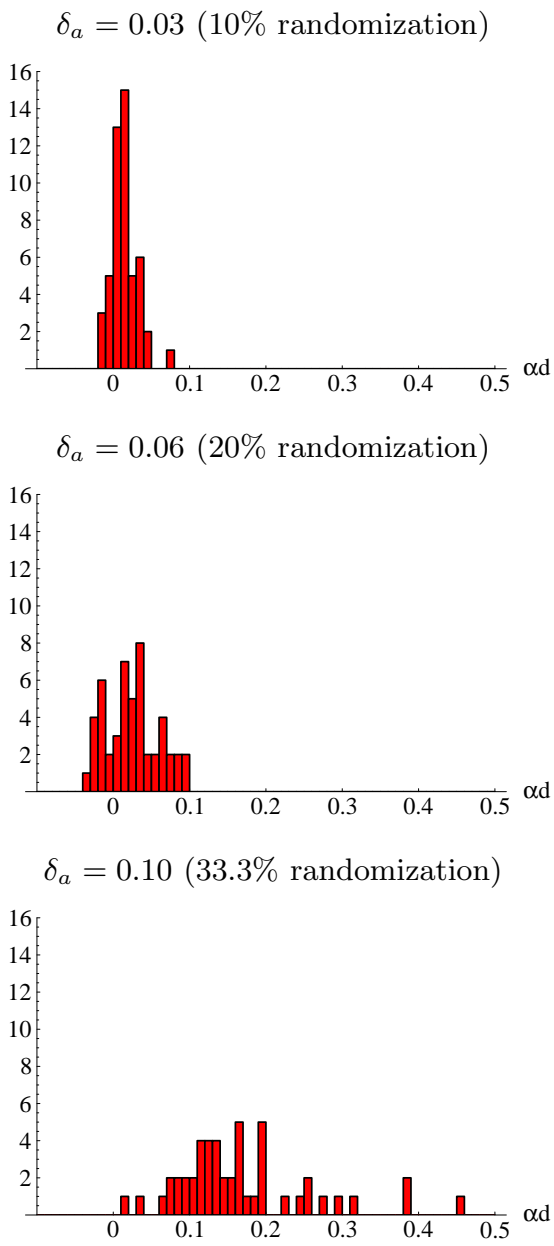

FIG. 5: Histograms of normalized intensity attenuation coefficients $\alpha d$ along the centre of the straight guide with disordered radius: 48 realizations with one-sided perturbations of normalized radius uniformly distributed on $\left[0, \delta_{a}\right]$.

matrix method [15], the Bloch modes of the crystal may be generated (see Appendix) and from these the reflection scattering matrix $\boldsymbol{R}_{\infty}$ for the semi-infinite crystal may be deduced. $\boldsymbol{R}_{\infty}$ is a function of wavenumber $k$, Bloch vector $\beta_{0}$ and material properties, including the cylinder radius $a$.

The field within the guide may be represented by a linear combination of left and right traveling plane waves

$$
V(x, y)=\sum_{p=-\infty}^{\infty}\left[f_{p}^{-} e^{-i \chi_{p}(x-h / 2)}+f_{p}^{+} e^{i \chi_{p}(x+h / 2)}\right] e^{i \beta_{p} y}
$$

where $\beta_{p}=\beta_{0}+2 \pi p / d$ and $\chi_{p}=\sqrt{k^{2}-\beta_{p}^{2}}$. In (6), phase origins are set at the boundaries $x= \pm h / 2$ of the crystal walls. In vector notation, with $\boldsymbol{f}_{ \pm}=\left[f_{p}^{ \pm}\right]$denoting vectors of plane wave field coefficients, and $\boldsymbol{P}=\operatorname{diag} \exp \left(i \chi_{p} h\right)$ characterizing plane wave propagation across the cavity, it follows that the consistency conditions for propagation are

$$
\boldsymbol{f}_{-}=\boldsymbol{R}_{\infty} \boldsymbol{P} \boldsymbol{f}_{+}, \quad \boldsymbol{f}_{+}=\boldsymbol{R}_{\infty} \boldsymbol{P} \boldsymbol{f}_{-} .
$$

These yield the dispersion relation

$$
\operatorname{det} \boldsymbol{G}\left(k, \beta_{0}\right)=0, \quad \text { where } \boldsymbol{G}=\boldsymbol{I}-\boldsymbol{R}_{\infty} \boldsymbol{P} \boldsymbol{R}_{\infty} \boldsymbol{P},
$$

which is solved for $\beta_{0}$ using standard numerical techniques. While these require the truncation of infinite series and, correspondingly, truncation of the dimensions of the matrices $\boldsymbol{R}_{\infty}$ and $\boldsymbol{P}$, the treatment is very robust, generating, for this configuration, propagation constants $\beta_{0}$ which converge to at least six significant figures for symmetrically truncated series containing five or more terms. Better than three significant figures of accuracy are available when 


$$
\delta_{a}=0.03(10 \% \text { randomization })
$$
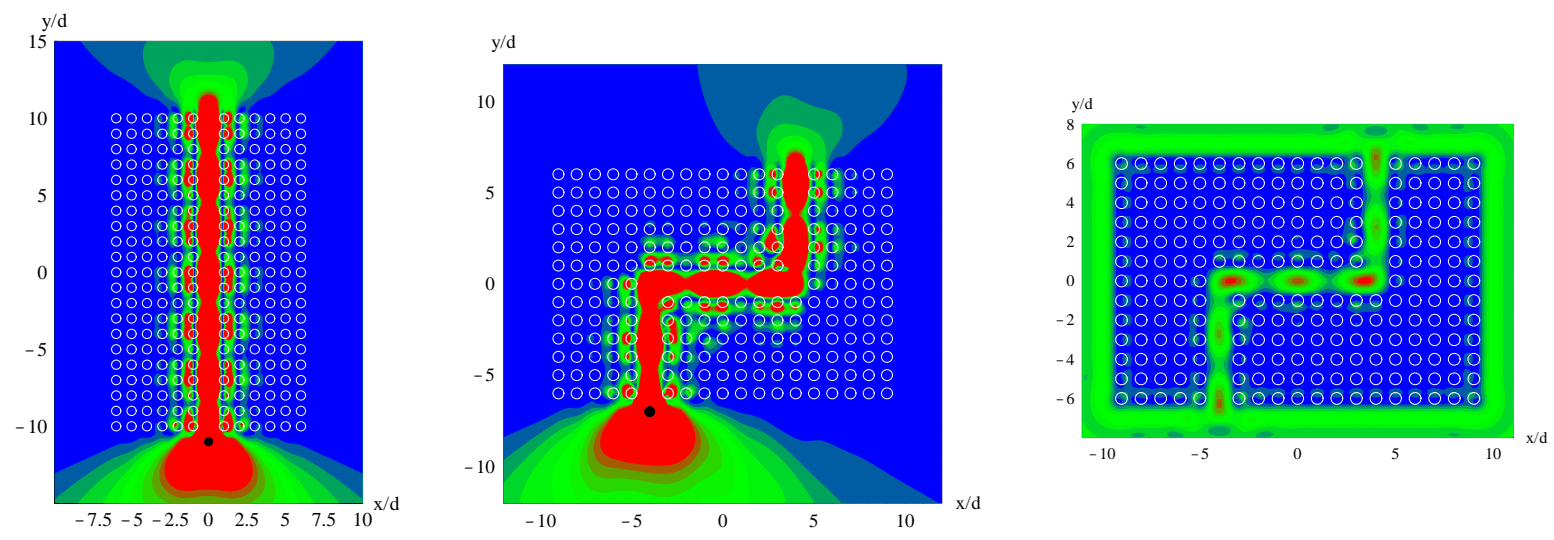

$$
\delta_{a}=0.06(20 \% \text { randomization })
$$
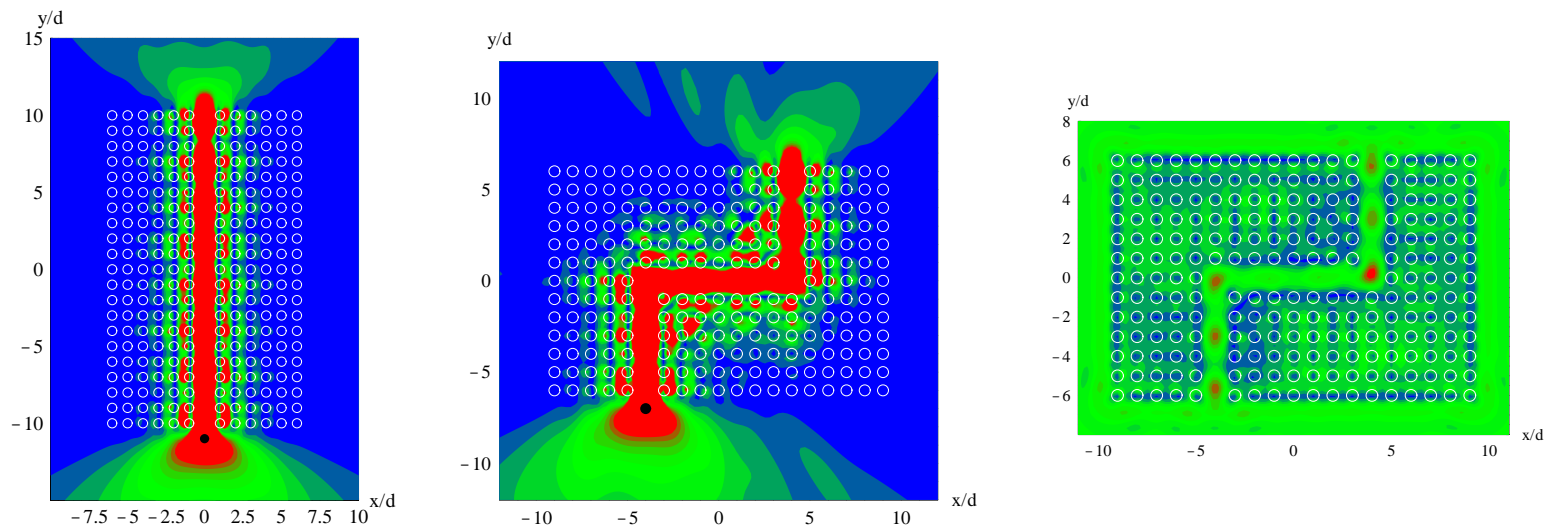

$\delta_{a}=0.10(33.3 \%$ randomization $)$
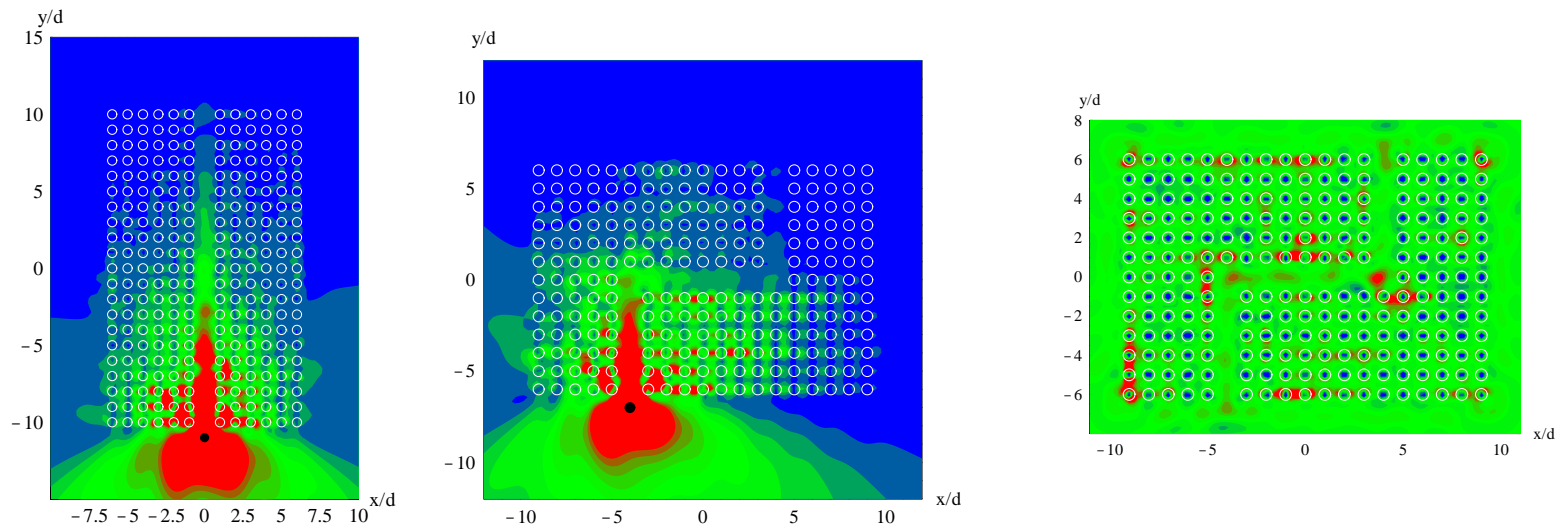

FIG. 6: (Color online) Uniform radius disorder: one-sided. Comparison of disorder effects in straight and bent guides: whole crystal. Left and centre: mean normalized intensity; right: mean normalized LDOS.

only the single zeroth order plane wave term is included, demonstrating that for a wavelength $\lambda=3.3 d$, which is long in comparison with the lattice constant, the diffraction problem is dominated by the specular plane wave. In this case, the dispersion relation can be simplified [15] to a simple scalar approximation

$$
\arg R_{\infty}=\cos ^{-1} \frac{\operatorname{Re} R_{00}}{\left|R_{00}\right|^{2}}=-\chi_{0} h
$$



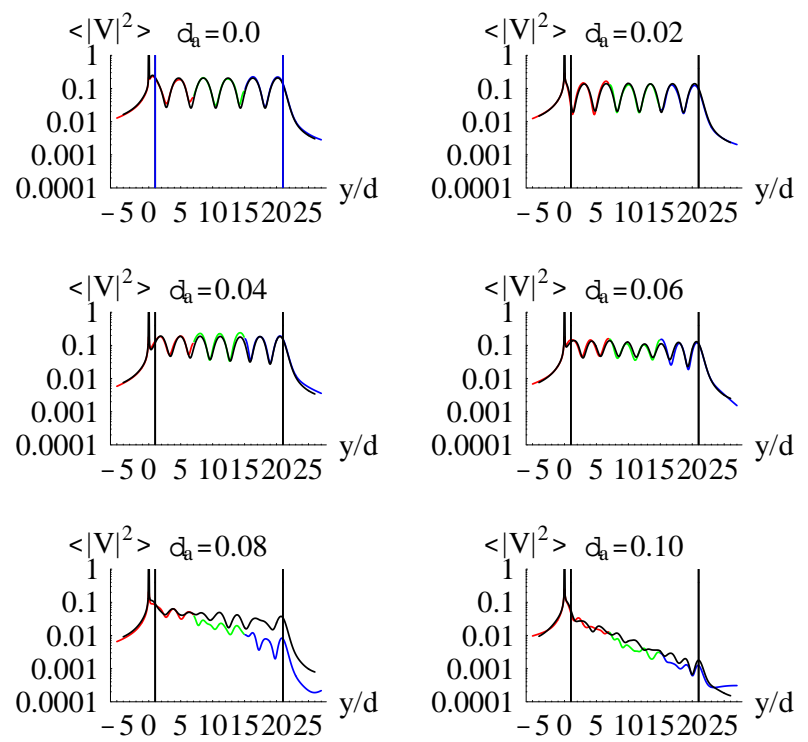

FIG. 7: (Color online) Uniform radius disorder: one-sided. Comparison of the mean normalized intensity in straight and bent guides with disordered radius at the same levels of randomization: centre of guide.

(a)

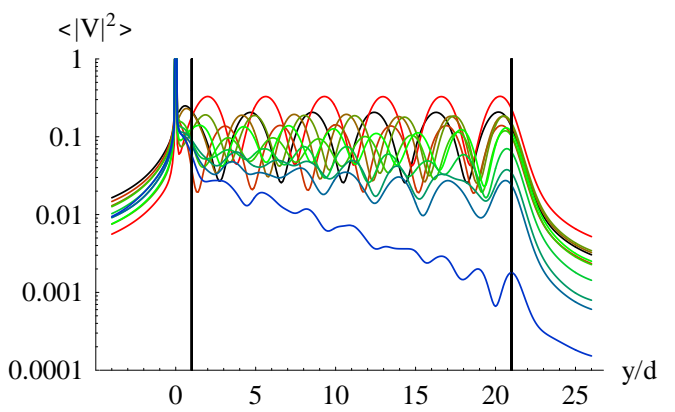

(b)

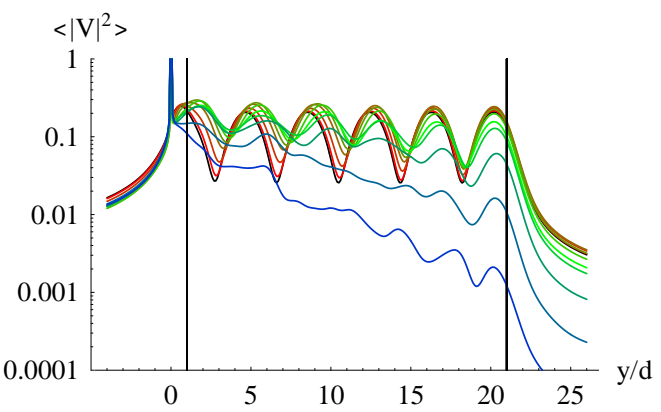

FIG. 8: (Color online) Combined plots of mean normalized intensity of straight guide with increasing disorder: centre of guide. (a) Case A1 (uniform radius disorder: one-sided). (b) Case A3 (uniform area disorder: two-sided).

where Re denotes the real part and $R_{00}$ denotes the specular (00) reflection coefficient of the cylinder gratings that constitute the semi-infinite crystals.

In Figure 9, the continuous curve shows the variation of the propagation constant $\beta_{0}$ (Eqs. (8) and (9)) with radius, while the superimposed points are derived from the data of Fig. 6 and Fig. 8, with abscissa values corresponding to the mean radius of the one-sided distribution $a / d=0.3+\delta_{a} / 2$. There is remarkable agreement between the two for $\delta_{a}<0.06(\langle a\rangle / d=0.33)$, after which the effects of the disorder become substantial, in keeping with the above discussion and the data of Fig. 4.

In contrast, cases A2 (double-sided uniform distribution of radius perturbations: $a_{\ell} / d$ uniformly distributed with $0.3-\delta_{a} \leq a_{\ell} / d \leq 0.3+\delta_{a}$ ) and A3 (double-sided uniform distribution of cylinder cross-sectional area perturbations $\left.\delta_{A}\right)$ yield quite different results. In the first case the mean change in fill factor is greatly reduced, and in the second it is zero. The results of both are similar-Fig. 8 suggests that we need quite large degrees of disorder or structures of different length to produce significant change in the beat length in these cases. We note that similar results were obtained for the bent guide. 


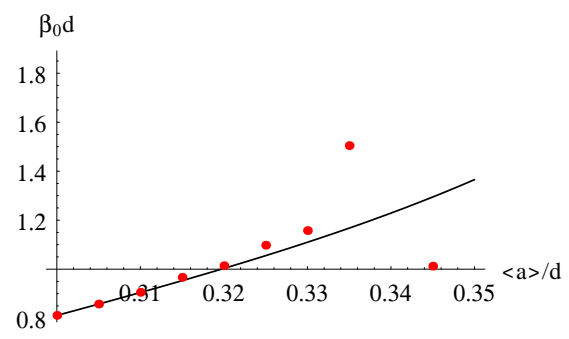

FIG. 9: Normalized propagation constant $\left(\beta_{0} d\right)$ versus normalized mean cylinder radius $\langle a\rangle / d$ for a disordered guide embedded in a finite cluster (isolated points), compared with that of a guide formed by a cavity between two semi-infinite crystals with no disorder and cylinder radii $\langle a\rangle$ (continuous curve). The finite cluster is subject to one-sided uniform radius disorder (case A1) yielding a propagation constant which increases with the degree of disorder (Fig 8(a)) and hence with the mean radius $\langle a\rangle$ of the cylinders: $\delta_{a}=0.01$ corresponds to $\langle a\rangle / d=0.305$ and $\delta_{a}=0.1$ corresponds to $\langle a\rangle / d=0.35$.
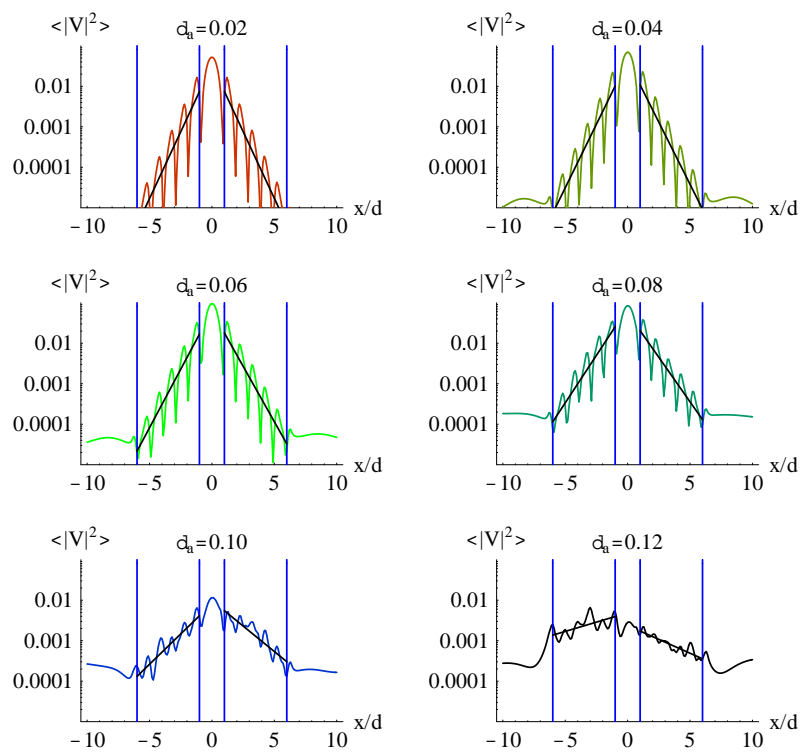

FIG. 10: (Color online) Uniform area disorder: two-sided. Logarithmic plots of mean normalized intensity at different levels of disorder in straight guide along cross-section cutting the crystal at the midpoint of the guide.

\section{TRANSITION TO TWO-DIMENSIONAL BEHAVIOUR}

Logarithmic normalized intensity plots along the channel suggest that attenuation along the channel is relatively small for low levels of disorder, but increases for $\delta_{a}>0.06$ (for this crystal). Correspondingly, with strong bandgap guiding for small disorder we expect strong decay of the field into the crystal interior. Figure 10 plots the normalized intensity for case A3 along a cross-section cutting the crystal halfway along the guide, for different levels of disorder. The four vertical lines in each plot indicate the positions of the outer layers of the crystal and the sides of the channel, with the channel itself in the centre and free space at either end. For $\delta_{a} \leq 0.06$ we again observe strong containment of the field in the channel - the linear fit indicates that decay into the crystal is exponential on average. For $\delta_{a}>0.06$ we observe that containment weakens and the quality of the linear fit deteriorates, indicating both decreasing attenuation into the crystal and a possible shift away from exponential (on average) decay.

The results described so far suggest that as disorder increases, in both the straight and bent guides, there is a transition from essentially one-dimensional behaviour along the guide to two-dimensional behaviour as the guiding breaks down. Linear fits of the logarithmic data allow us to compute, for each realization, normalized attenuation coefficients along the channel for both the straight and bent guides, and along cross-sections cutting the channel for the straight guide. Figure 11(a) gives scatter plots for the normalized attenuation coefficients along the channel for individual realizations of the straight and bent guides, against level of disorder. The means of these coefficients are indicated by corresponding lines. Again we observe that the behaviour is not strongly affected for $\delta_{a} \leq 0.06$. 

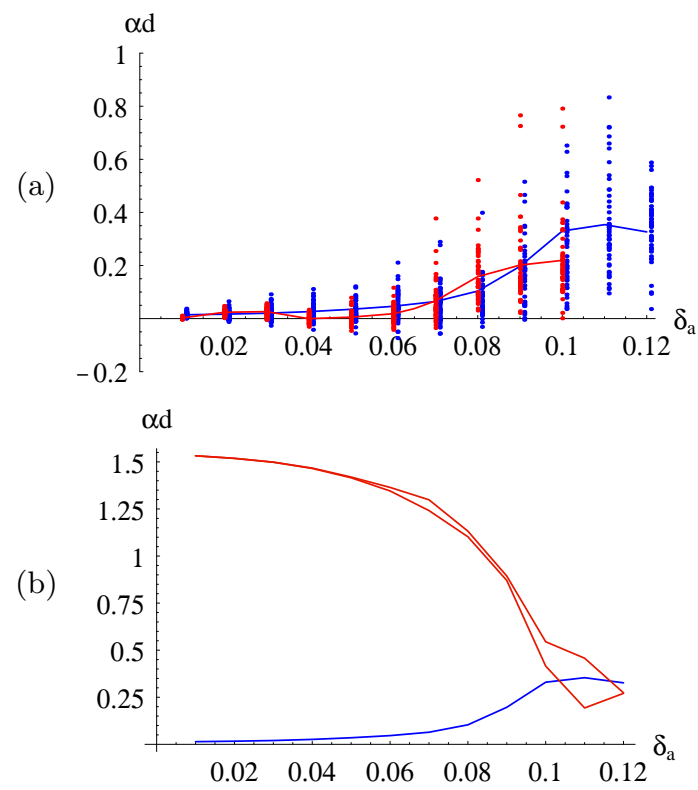

FIG. 11: (Color online) (a) Scatter plot of normalized attenuation coefficients $\alpha d$ along the guide for individual realizations of crystals. Coefficients for the straight guide with disordered radius (one-sided) are slightly offset to the left of those for the bent guide with disordered area (two-sided). The mean coefficients for each level of randomization are joined by a line, for ease of inspection. (b) Mean normalized attenuation coefficients for the straight guide with disordered area, two-sided. The lower line gives mean coefficients for the field along the middle of the guide. The two upper lines give mean coefficients along cross-sections to the left and right of the midpoint of the guide.

Figure 11(b) plots just the means of the normalized attenuation coefficients for realizations of the straight guide, both along the channel and along a cross-section through the centre of the cluster, perpendicular to the channel. The wide separation between these values for $\delta_{a} \leq 0.06$ is indicative of 1-dimensional guiding behaviour. However, the decreasing separation for $0.06 \leq \delta_{a} \leq 0.1$ indicates a transition from 1D to $2 \mathrm{D}$ behaviour characterizing the closure of the guide, this being achieved as the curves meet for $\delta_{a}>0.1$. Results for uniform perturbations of index (case N1) are very similar.

\section{DISCUSSION AND CONCLUSIONS}

The guides studied were robust with respect to levels of disorder (uniformly distributed random perturbations of radius or index) of up to about $20 \%$, even when guides contain bends. However, biases in distribution, while not affecting the fact of guiding (at least for small levels of disorder), can have major effects on optical characteristics of the guide (eg beat length). At least in some cases it appears that, to a first order of approximation, the optical characteristics of the guide remain unaffected by the existence of bends in the guide. For sufficiently high levels of disorder, the breakdown of guiding manifests itself as a transition from one-dimensional to two-dimensional behaviour.

We note that the analysis given here does not include some of the effects that are important in practical devices. For example, the cylinders are assumed to be of infinite length, and the propagation is assumed to be exclusively in the plane orthogonal to the cylinder axes. In practice, the devices include cylinders of finite length, with the confinement in this direction typically provided by total internal reflection. Thus, the analysis ignores the losses of light that has been scattered by the disorder to propagate in directions where the total internal reflection condition is violated, so that it can escape the waveguide.

Despite these caveats, these results appear encouraging for the practical application of two-dimensional photonic crystal waveguides, in the sense that moderate errors in radius and refractive index appear to have a relatively small effect on guiding.

Acknowledgements. The authors wish to acknowledge both the direct support of the Australian Research Council and its support for the Centre of Excellence for Ultra-wide-bandwidth Devices in Optical Systems. The authors also acknowledge the support of the Australian Centre for Advanced Computing and Communications (ac3) and the Australian Partnership for Advanced Computing (APAC) National Facility. 


\section{APPENDIX}

Here, we outline the formation of the reflection matrix $\boldsymbol{R}_{\infty}$ from the computation of the Bloch modes of the crystal using a transfer matrix method. In this, we characterize the properties of each constituent cylinder grating with reflection $\boldsymbol{R}$ and transmission $\boldsymbol{T}$ scattering matrices. As in $[15,16]$, the matrices are indexed by integers $p$ and $q$, e.g. $R_{p q}$, denoting respectively input and output channels that correspond to directions given by wave numbers $\beta_{p}$ and $\beta_{q}$. While the reflection and transmission scattering matrices may be computed with a variety of techniques, we use multipole methods [16] for reasons of accuracy and efficiency.

Following the treatment of [15], the fields between the layers of the crystal are written in plane wave expansions

$$
V(x, y)=\sum_{p=-\infty}^{\infty}\left[g_{p}^{-} e^{-i \chi_{p} x}+g_{p}^{+} e^{i \chi_{p} x}\right] e^{i \beta_{p} y} .
$$

Using the Bloch condition for field quasiperiodicity between grating layers, we form the eigenvalue equation

$$
\mathcal{T}\left(\begin{array}{l}
\boldsymbol{g}_{+} \\
\boldsymbol{g}_{-}
\end{array}\right)=\mu\left(\begin{array}{l}
\boldsymbol{g}_{+} \\
\boldsymbol{g}_{-}
\end{array}\right)
$$

from which the Bloch factors (eigenvalues) are obtained. Here,

$$
\mathcal{T}=\left(\begin{array}{cc}
T-R T^{-1} R & R T^{-1} \\
-T^{-1} R & T^{-1}
\end{array}\right)
$$

is the interlayer translation operator.

Bloch modes occur in forward/backward propagating pairs, leading to a natural partitioning of the mode set. For rectangular lattices, it can be shown that if $\mu$ is an eigenvalue corresponding to a forward propagating mode denoted by the pair $\left(\boldsymbol{g}_{+}, \boldsymbol{g}_{-}\right)$, then the corresponding backward propagating state has eigenvalue $\mu^{-1}$ and eigenvector pair $\left(\boldsymbol{g}_{-}, \boldsymbol{g}_{+}\right)$.

For a semi-infinite crystal, for which there is no rear surface from which to generate backward propagating modes, the propagation problem can be solved exclusively in terms of forward propagating states. Introducing matrices $\boldsymbol{G}_{ \pm}$, the columns of which comprise the eigenvectors $\boldsymbol{g}_{ \pm}$of the forward modes, we can write the incident plane wave field $\boldsymbol{\delta}$ and the reflected field $\boldsymbol{R}$ at the interface of the semi-infinite crystals as a linear combination of the forward propagating Bloch modes

$$
\left(\begin{array}{l}
\delta \\
r
\end{array}\right)=\left(\begin{array}{l}
G_{+} \\
G_{-}
\end{array}\right) c
$$

Eliminating the coefficients $\boldsymbol{c}$ leads naturally to the definition of $\boldsymbol{R}_{\infty}$ :

$$
\boldsymbol{r} \stackrel{\text { def }}{=} \boldsymbol{R}_{\infty} \boldsymbol{\delta}, \quad \text { where } \boldsymbol{R}_{\infty}=\boldsymbol{G}_{-} \boldsymbol{G}_{+}^{-1} .
$$

[1] J. D. Joannopoulos, S. Fan, A. Mekis, and S. G. Johnson, Photonic crystals and light localization in the 21st Century, 1 (2001).

[2] E. Yablonovitch, Phys. Rev. Lett. 58, 2059 (1987).

[3] S. John, Phys. Rev. Lett. 58, 2486 (1987).

[4] A. Mekis, J. C. Chen, I. Kurland, S. Fan, P. R. Villeneuve, and J. D. Joannopoulos, Phys. Rev. Lett. 77, 3787 (1996).

[5] S.-Y. Lin, E. Chow, V. Hietala, P. R. Villeneuve, and J. D. Joannopoulos, Science 282, 274 (1998).

[6] J. Yonekura, M. Ikeda, and T. Baba, J. Lightwave Technol. 17, 1500 (1999).

[7] S. Fan, P. R. Villeneuve, J. D. Joannopoulos, and H. A. Haus, Opt. Express 3, 4 (1998).

[8] G. Parker and M. Charlton, Physics World 13, 29 (2000).

[9] S. John and M. Florescu, J. Opt. A. Pure Appl. Opt. 3, S103 (2001).

[10] L. C. Botten, R. C. McPhedran, N. A. Nicorovici, A. A. Asatryan, C. M. de Sterke, P. A. Robinson, K. Busch, G. H. Smith, and T. N. Langtry, in PIER Special Issue on Electromagnetic Applications of Photonic Band Gap Materials and Structures (in press).

[11] M. H. Kalos and P. A. Whitlock, Monte Carlo Methods (John Wiley and Sons, New York, 1986).

[12] A. Asatryan, K. Busch, R. C. McPhedran, L. C. Botten, C. M. de Sterke, and N. A. Nicorovici, Waves Random Media 13, $9(2003)$. 
[13] A. Asatryan, K. Busch, R. C. McPhedran, L. C. Botten, C. M. de Sterke, and N. A. Nicorovici, Phys. Rev. E 63, 46612 (2001).

[14] R. Balian and C. Bloch, Annals of Physics 64, 271 (1971).

[15] L. C. Botten, N. A. Nicorovici, R. C. McPhedran, C. M. de Sterke, and A. A. Asatryan, Phys. Rev. E 64, 046603 (2001).

[16] L. C. Botten, N. A. Nicorovici, A. A. Asatryan, R. C. McPhedran, C. M. de Sterke, and P. A. Robinson, J. Opt. Soc. Am. A 17, $2165(2000)$. 


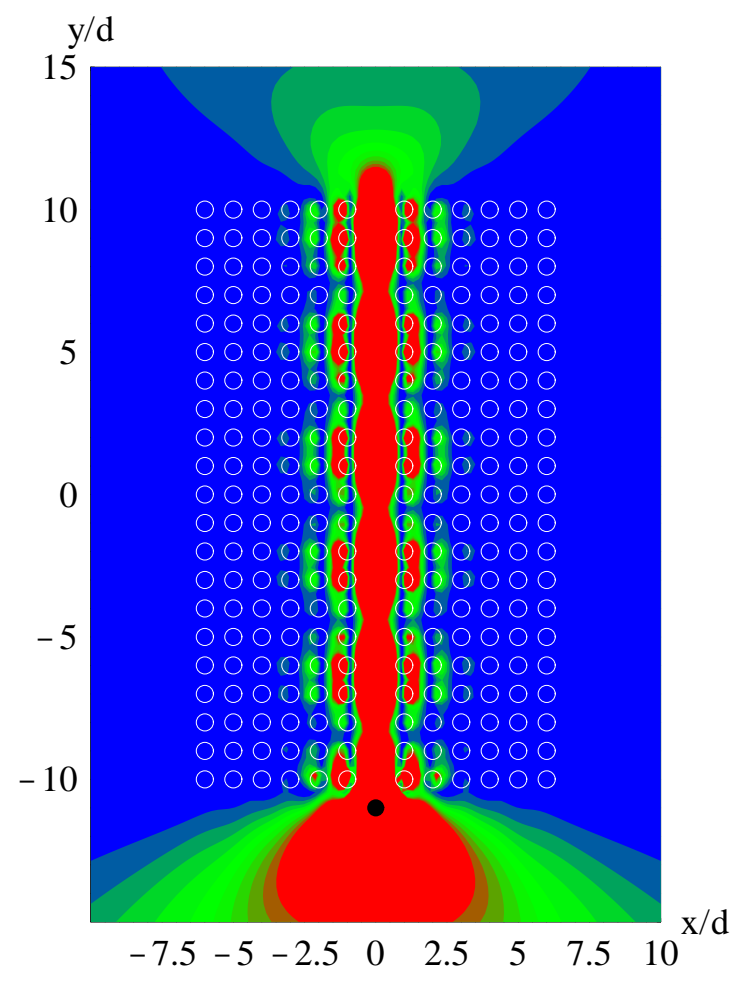

Figure 1a 


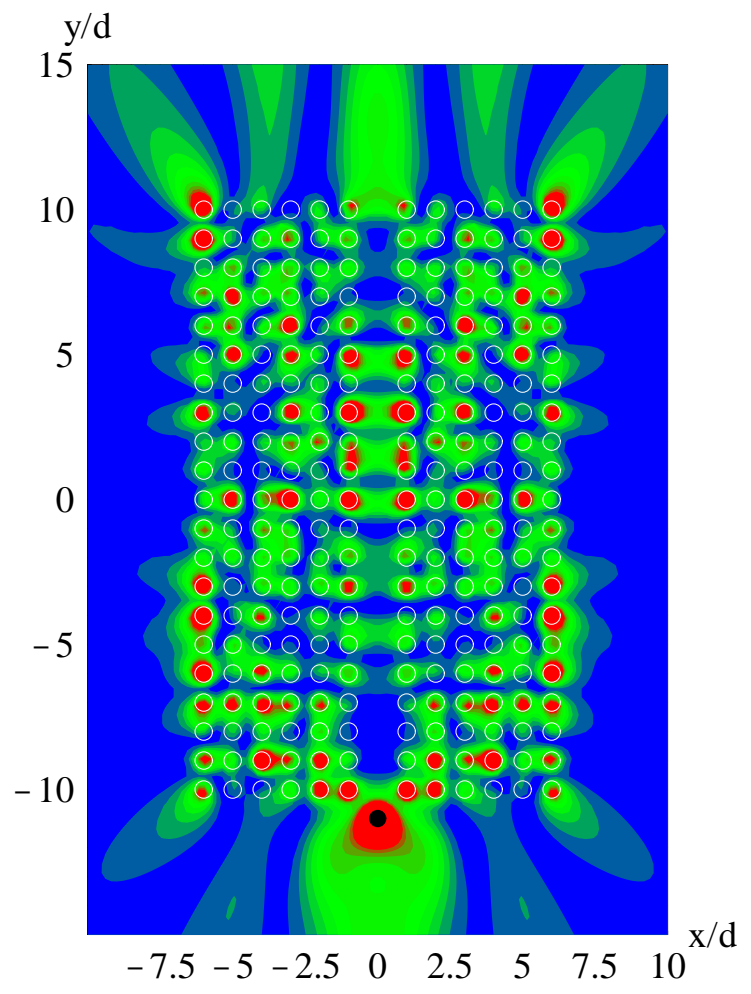

Figure $1 b$ 


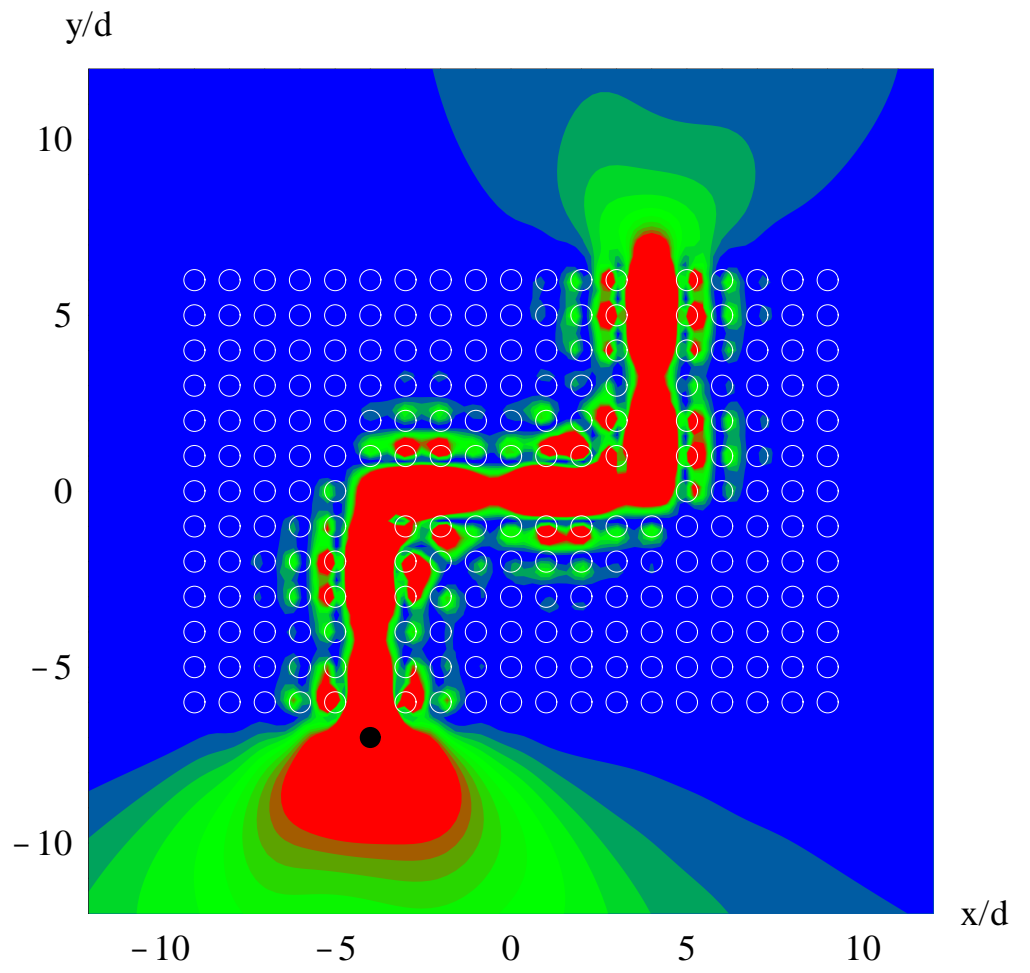

Figure 1c 